\title{
A Simple Reaction Time Scale for Under-Resolved Fire Dynamics
}

\author{
RANDALL McDERMOTT ${ }^{1}$, KEVIN McGRATTAN ${ }^{1}$, and JASON FLOYD ${ }^{2}$ \\ ${ }^{1}$ Building and Fire Research Laboratory \\ National Institute of Standards and Technology (NIST) \\ Gaithersburg, Maryland, USA \\ ${ }^{2}$ Hughes Associates, Inc. \\ Baltimore, Maryland, USA
}

\begin{abstract}
A reaction time scale model is developed for use in the eddy dissipation concept (fast chemistry limit) closure of the mean chemical source term in large-eddy simulation of fires. The novel aspect of the model is to consider a scaling regime for coarse mesh resolution based on buoyant acceleration. The model computes local time scales for diffusion, turbulent advection, and buoyant acceleration and takes the minimum of these as the local mixing time. The new model is implemented in the Fire Dynamics Simulator (FDS) and tested by comparing flame height predictions to the Heskestad correlation.
\end{abstract}

KEYWORDS: modeling, large-eddy simulation, eddy dissipation concept, flame height, buoyancy.

\section{NOMENCLATURE}

\begin{tabular}{|c|c|c|c|}
\hline$C=1.5$ & Kolmogorov constant & $\delta t$ & simulation time step (s) \\
\hline$C_{s}$ & Smagorinsky constant & $\varepsilon$ & dissipation rate $\left(\mathrm{m}^{2} / \mathrm{s}^{3}\right)$ \\
\hline$c_{p}$ & heat capacity $(\mathrm{kJ} / \mathrm{kg} \cdot \mathrm{K})$ & $\kappa$ & wavenumber $(1 / \mathrm{m})$ \\
\hline$D$ & pool fire diameter $(\mathrm{m})$ & $\mu$ & dynamic viscosity $(\mathrm{kg} / \mathrm{m} \cdot \mathrm{s})$ \\
\hline$D^{*}$ & characteristic length scale (m) & $\eta$ & Kolmogorov length scale (m) \\
\hline$F, \mathrm{O}_{2}, P$ & fuel, oxygen, products & $\phi$ & arbitrary scalar quantity \\
\hline$g$ & acceleration due to gravity $\left(\mathrm{m} / \mathrm{s}^{2}\right)$ & $\rho$ & mass density $\left(\mathrm{kg} / \mathrm{m}^{3}\right)$ \\
\hline$k_{s g s}$ & subgrid-scale kinetic energy $\left(\mathrm{m}^{2} / \mathrm{s}^{2}\right)$ & $\tau$ & mixing time scale (s) \\
\hline$L_{f}$ & flame length (m) & subscripts & \\
\hline$\dot{m}_{\alpha}^{\prime \prime \prime}$ & chemical source term $\left(\mathrm{kg} / \mathrm{s} \cdot \mathrm{m}^{3}\right)$ & 0 & background \\
\hline$\dot{Q}$ & fire heat release rate $(\mathrm{kW})$ & chem & chemistry \\
\hline$\dot{Q}^{\prime \prime \prime}$ & heat release rate per unit volume $\left(\mathrm{kW} / \mathrm{m}^{3}\right)$ & $d$ & diffusion \\
\hline$Q^{*}$ & fire Froude number & $F$ & fuel \\
\hline$r, x, z$ & space $(m)$ & flame & flame \\
\hline$s$ & mass stoichiometric coefficient of oxygen & $g$ & gravitational acceleration \\
\hline$S_{i j}$ & strain rate tensor component $(1 / \mathrm{s})$ & Grav & Gravitational \\
\hline$|S|$ & strain rate magnitude $(1 / \mathrm{s})$ & $i, j$ & spatial indices \\
\hline $\mathrm{Sc}_{t}, \operatorname{Pr}_{t}$ & turbulent Schmidt and Prandtl numbers & sgs & subgrid-scale \\
\hline$t$ & time $(\mathrm{s})$ & Smag & Smagorinsky \\
\hline$T$ & temperature $(\mathrm{K})$ & $t$ & turbulent \\
\hline$u_{i}$ & velocity component $(\mathrm{m} / \mathrm{s})$ & $u$ & advection \\
\hline$Y_{\alpha}$ & mass fraction of species $\alpha$ & $\alpha$ & species index \\
\hline Greek & & $\infty$ & ambient \\
\hline$\Delta$ & filter width (m) & special ope & rators \\
\hline$\Delta H_{F}$ & heat of combustion $(\mathrm{kJ} / \mathrm{kg})$ & $\bar{\phi}$ & cell mean (filtered scalar) \\
\hline$\delta x$ & grid spacing $(\mathrm{m})$ & $\tilde{\phi} \equiv \overline{\rho \phi} / \bar{\rho}$ & Favre-filtered (mass weighted) \\
\hline
\end{tabular}

\section{INTRODUCTION}

In order of increasing fidelity, computational fluid dynamics (CFD) models for fire may be subdivided into: steady state Reynolds-Averaged Navier-Stokes (RANS), unsteady RANS (URANS), large-eddy simulation (LES), and direct numerical simulation (DNS). Only recently has LES become practical for engineering calculations, and generally such LES require relatively simple and robust submodels. A simple LES submodel 
for combustion is the subject of this paper. Presently, DNS is too expensive for general fire safety engineering problems but does find use in fire research applications. Without getting sidetracked into details, here we consider URANS to live in the gray area between RANS and LES. The discussion to follow, though principally aimed at LES, may also apply to sufficiently resolved URANS models.

The development of the LES equations involves introduction of an artificial (nonphysical) parameter, the filter width, $\Delta$, into the model system $[1,2]$. In practical LES, $\Delta$ is roughly equivalent to the mesh spacing and second-order numerical accuracy is the best one can expect. The unfortunate result is that numerical errors and physical modeling errors are difficult to untangle. But all hope is not lost. For, in any real scenario, only a statistical equivalence between the model and reality is achievable [2]. Given this state of affairs, we must pay close attention to how our submodels behave as the filter width (grid size) changes. In particular, it is vital that the model is convergent in the sense that as $\Delta \rightarrow 0$ we recover a DNS formulation.

At the opposite end of the spectrum, practical engineering calculations often require coarse grids and submodels designed for fine grids may become inappropriate. For example, if-as is often the case in LES-the submodel assumes the filter width lies within the inertial subrange of a sufficiently high Reynolds number (Re) flow, then coarser filter widths will likely lead to poor results. To address this problem, the practitioner either needs to test the resolution quality [3] of the calculation and refine the mesh if necessary, or they need to instead use a more flexible submodel which is accurate for the specified filter width. The work in this paper focusses on the second approach specifically for the reaction submodel in the Fire Dynamics Simulator (FDS) [4-6] which is a CFD fire model based on LES.

For fire, the heat releasing reactions are generally controlled by the rate of mixing between the fuel and the oxidant. For most practical purposes, the chemistry may be considered infinitely fast (though soot generation, which affects radiation, is a slow chemical process). Estimating the mean reaction rate then involves some estimate of the mixing time scale. Numerous approaches for modeling the mean reaction rate and mixing time scale exist. The reader is referred to Fox [7], Peters [8], and Poinsot and Veynante [9] for excellent reviews. One of the earliest and simplest ideas for modeling turbulent diffusion flames is the eddy dissipation concept (EDC) of Magnussen and Hjertager [10]. In this paper, we consider fast chemistry and basically extend the cascade model of Ertesvåg and Magnussen [11] to coarse mesh spacing such that the filter width may lie outside the inertial subrange.

In FDS version 5, the EDC time scale is based on the turbulent diffusivity alone and the proportionality constant in the model is tuned for a specific resolution. Further, limiters on the heat release rate (determined from trial and error) are required to maintain stability and to achieve reasonable flame heights for different grid resolutions. In some cases, careful examination of the results shows the heat release limiters are invoked a high percentage of the time, effectively becoming the model for combustion. Recent advancements to FDS, which include addition of the dynamic Smagorinsky turbulence model [12,13], addition of total variation diminishing (TVD) scalar transport schemes [14], restoration of the baroclinic torque, and the improvements to the reaction model discussed in this paper, have corrected the situation: the submodels are suitable across a broad range of grid spacings and physically-based limiters need only be applied in rare circumstances.

\section{FORMULATION}

Full details of the FDS formulation and numerical procedures may be found in the FDS Technical Reference Guide [4]. It is useful, however, to briefly highlight components of the model which are important for mixing and reaction.

\section{Filtered Fields}

The LES equations are derived by applying a low-pass filter, parameterized by a width $\Delta$, to the transport equations for mass, momentum and energy. For our purposes, it is sufficient to think of the filtered fields in the LES equations as cell means. For example, in 1D the filtered density for a cell of width $\Delta$ is

$\bar{\rho}(x, t)=\frac{1}{\Delta} \int_{x-\Delta / 2}^{x+\Delta / 2} \rho(r, t) \mathrm{d} r$. 
It is also useful to define a Favre-filtered field (see e.g. [8]) for an arbitrary scalar $\phi$ by $\tilde{\phi} \equiv \overline{\rho \phi} / \bar{\rho}$.

In FDS, the filter width $\Delta$ is equivalent to the local cell size $\delta x$ and is a key parameter in the submodels for the turbulent viscosity and the reaction time scale developed in this paper.

\section{Species Transport}

FDS solves transport equations for Favre-filtered species mass fractions $\tilde{Y}_{\alpha}$,

$\frac{\partial \bar{\rho} \tilde{Y}_{\alpha}}{\partial t}+\frac{\partial\left(\bar{\rho} \tilde{Y}_{\alpha} \tilde{u}_{i}\right)}{\partial x_{i}}=\frac{\partial}{\partial x_{i}}\left(\left[\frac{\mu+\mu_{t}}{\mathrm{Sc}_{t}}\right] \frac{\partial \tilde{Y}_{\alpha}}{\partial x_{i}}\right)+\overline{\dot{m}_{\alpha}^{\prime \prime \prime}}$,

where $\bar{\rho}$ is the filter density and $\tilde{u}_{i}$ is a Favre-filtered velocity component. The turbulent viscosity $\mu_{t}$, which we obtain from the dynamic Smagorinsky model $[12,13]$, plays a particularly important role in the model, controlling the level of large scale dynamics as well as subgrid scale mixing. The turbulent Schmidt number, $\mathrm{Sc}_{t}$, is set to 0.5 [15]. The molecular viscosity, $\mu$, is a function of species concentration and temperature. The numerical methods used to solve Eq. 2 are explicit and generally second-order in space and time.

\section{Mean Chemical Source Term}

One of the most challenging problems in modeling turbulent reacting flows is the closure of the mean chemical source term, $\overline{\dot{m}_{\alpha}^{\prime \prime \prime}}$, in Eq. 2. Many approaches exist for handling this term [7-9]. Here, we consider the limiting case of infinitely fast chemistry for nonpremixed flames. The rate of reaction is therefore controlled by the rate of mixing between fuel and oxygen which is usually dominated by turbulence. The fuel, oxidant, and products are tracked as lumped species and we consider the following simplified reaction (fuel plus oxygen goes to products),

$\mathrm{F}+\mathrm{sO}_{2} \longrightarrow \mathrm{P}$

where $s$ is the mass stoichoimetric ratio of oxygen to fuel. The rate of reaction is modeled by the eddy dissipation concept (EDC) of Magnussen and Hjertager $[9,10]$,

$\overline{\dot{m}_{F}^{\prime \prime \prime}}=\bar{\rho} \frac{\mathrm{d} \tilde{Y}_{F}}{\mathrm{~d} t}=-\frac{\bar{\rho} \min \left(\tilde{Y}_{F}, \tilde{Y}_{O} / s\right)}{\tau_{m i x}}$.

The reaction rate is proportional to the local stoichiometric concentration of fuel. All of the physics is then buried in the reaction time scale (or mixing time), $\tau_{\text {mix }}$. Specification of the mixing time over a broad range of filter widths is the main focus of this paper and is discussed in detail below.

In the FDS combustion model, each cell is thought of as a batch reactor with a residence time $\delta t$, the time increment in the simulation. The faster the reactants mix (the smaller $\tau_{m i x}$ ) the more heat is released. FDS uses a time splitting for chemistry where Eq. 4 is integrated after the species transport step. The mean rate of heat release per unit volume over the time step is then given by

$$
\begin{aligned}
\dot{Q}^{\prime \prime \prime} & =-\frac{\Delta H_{F}}{\delta t} \int_{0}^{\delta t} \overline{\dot{m}_{F}^{\prime \prime \prime}} \mathrm{d} t, \\
& =\frac{\Delta H_{F}}{\delta t} \bar{\rho} \min \left(\tilde{Y}_{F}(0), \tilde{Y}_{O}(0) / s\right)\left[1-\exp \left(-\delta t / \tau_{m i x}\right)\right],
\end{aligned}
$$

where $\Delta H_{F}$ is the heat of combustion. The initial fuel and oxidizer mass fractions, $\tilde{Y}_{F}(0)$ and $\tilde{Y}_{O}(0)$, are taken from the start of the chemistry step (i.e., after the transport step). It it evident from Eq. 5 that $\tau_{m i x}$ is the time required to mix roughly two thirds of the reactants within a cell. 


\section{Flame Suppression}

Note that the combustion reaction may be suppressed by an extinction model, as discussed in Vaari et al. [16]. If the potential heat release of the local fuel/air mixture is not enough to lift the mixture temperature above an empirically determined lower limit [17], then combustion is disallowed. That is, $\overline{\dot{m}_{F}^{\prime \prime \prime}}$ is set to zero.

\section{REACTION TIME SCALE MODEL}

In the fast chemistry limit, our task is to provide an expression for the mixing time based on the local state of the flow field. The basic idea behind the model we propose here is to consider the three physical processes of diffusion, subgrid-scale (SGS) advection, and buoyant acceleration and to take the fastest of these processes (locally) as the controlling flow time scale.

As noted in the introduction, it is important to consider the behavior of a model as the filter width varies. The mixing times for diffusion, SGS advection, and buoyant acceleration scale differently with filter width and if we look to the limits of the filter scales an interesting picture emerges. Referring to Fig. 1, let us move from left to right along the horizontal axis following the thick black line which represents our time scale model for a hypothetical flow condition. First, notice that the reaction time scale must be greater than or equal to the chemical time scale which, though small, is finite. At a slightly larger scale we expect the mixing time to vary as the square of the filter width because the mixing is controlled by molecular diffusion. In this regime, denoted $\tau_{d}$, the numerical solution is a DNS and this scaling law is valid while $\Delta$ is less than the Kolmogorov scale, $\eta$, the length scale of the smallest turbulent eddies. For a sufficiently high Reynolds number flow (such that an inertial subrange exists), as the filter width increases beyond the Kolmogorov scale we encounter a regime, marked $\tau_{u}$, where turbulent advection controls the rate of mixing and the mixing time varies as the two thirds power of the filter width [1] (we will see why later). This is the regime where most LES submodels are valid.

Now let us imagine what should happen to the mixing time as the filter width increases beyond the inertial subrange to a length scale larger than the height of the flame itself (actually a possibility in wildfire modeling). We would not expect the inertial range scaling to continue up through the so-called "energycontaining" range of turbulent length scales. Rather, for fires-which are generally buoyancy-driven flows-we expect buoyant acceleration to control the mixing at these relatively coarse scales. A time scale based on a constant acceleration goes as the square root of the filter width, as shown by the regime marked $\tau_{g}$ in the diagram. This shift in scaling may appear minor given the log-log nature of the plot. But, as we will see, the effect of the acceleration-based time scale is indeed significant for large cell sizes. Finally, note that the flame height presents a limit to the reaction time scale, here denoted $\tau_{\text {flame }}$, since all fuel must be consumed within a single cell.

Of course, the relative importance of the physical processes will depend on the flow. For example, if gravity is weak the $\tau_{g}$ line shifts up and may not affect the reaction time before the flame time scale is reached. If the flow is highly turbulent, the inertial range scaling may be more dominant, which would be indicated by a lowering of the $\tau_{u}$ line. Or, for highly turbulent jet flames $\tau_{\text {flame }}$ may be reached before the acceleration time scale has any effect. Perhaps more typical for low strain fires, if an inertial subrange does not exist (if the Reynolds number is too low relative to the Froude number), then the $\tau_{u}$ line in Fig. 1 moves up out of the picture and we are left with diffusion and buoyancy to control the mixing.

The bold solid line in Fig. 1 is mathematically represented by

$\tau_{\text {mix }}=\max \left(\tau_{\text {chem }}, \min \left(\tau_{d}, \tau_{u}, \tau_{g}, \tau_{\text {flame }}\right)\right)$.

In the test cases presented later in this work, we take chemistry to be infinitely fast and our grid spacings stay within a quarter of the minimum flame dimension. For our purposes, therefore, both $\tau_{\text {chem }}$ and $\tau_{\text {flame }}$ may be ignored.

The mathematical details of the submodels are as follows: 


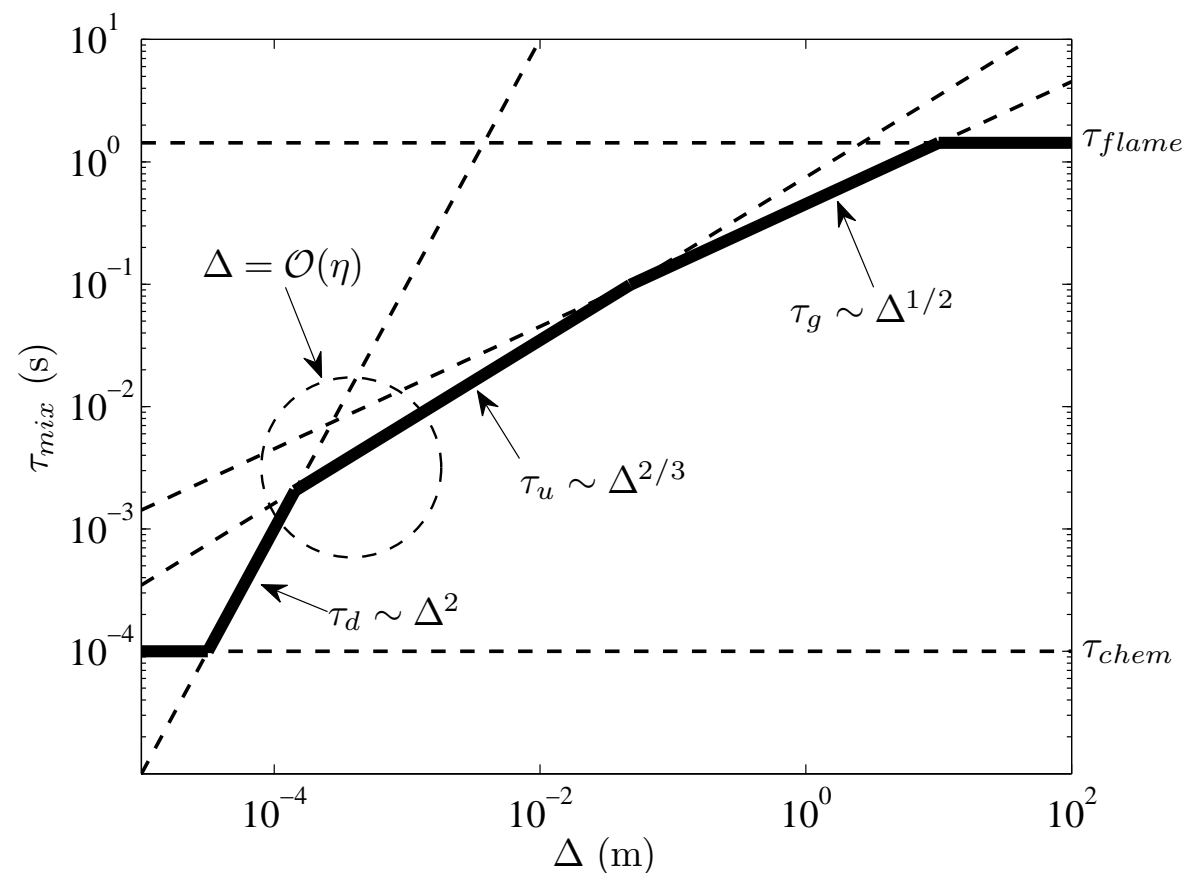

Fig. 1. Reaction time scale model.

$$
\begin{aligned}
& \tau_{d}=\frac{\mathrm{Sc}_{t} \overline{\mathrm{\rho}} \Delta^{2}}{\mu+\mu_{t}}, \\
& \tau_{u}=\frac{\Delta}{\sqrt{2 k_{s g s}}}, \\
& \tau_{g}=\sqrt{2 \Delta / g} .
\end{aligned}
$$

Note that $k_{s g s}$ is the unclosed subgrid kinetic energy per unit mass which is discussed further below. The acceleration time scale $\tau_{g}$ is the time required to travel a distance $\Delta$ starting from rest under a constant acceleration, $g=9.81 \mathrm{~m} / \mathrm{s}^{2}$.

Remark The turbulent viscosity is included in the diffusion time scale in Eq. 7 and the reader may wonder why the diffusion time scale does not then also account for turbulent advection. This idea is reasonable and in fact FDS 5 uses such a model. The shortcoming of the approach is that a proportionality constant is required in the expression and the correct diffusion behavior is then not recovered as $\Delta \rightarrow 0$. In FDS 5 , the model constant used in front of Eq. 7 is 0.1 , so for our proposed model $\tau_{d}>\tau_{u}$ in the subgrid advection regime. By including the turbulent viscosity in $\tau_{d}$, however, the diffusion time scale helps bridge the gap between dissipation and inertial range scaling.

\section{Estimating the Subgrid Kinetic Energy}

If the mixing is controlled by unresolved turbulent advection we presume that an inertial subrange of length scales exists and that the filter width lies within this range. The subgrid kinetic energy may then be estimated by integrating a Kolmogorov spectrum [1] from the filter wavenumber $(\kappa=\pi / \Delta)$ to the smallest possible length scales (highest wavenumbers), 


$$
\begin{aligned}
k_{s g s} & =C \varepsilon^{2 / 3} \int_{\pi / \Delta}^{\infty} \kappa^{-5 / 3} \mathrm{~d} \kappa, \\
& =C \varepsilon^{2 / 3} \frac{3}{2}\left(\frac{\Delta}{\pi}\right)^{2 / 3},
\end{aligned}
$$

where $C=1.5$ is the Kolmogorov constant [1] and $\varepsilon$ is the kinetic energy dissipation rate (which requires closure, as discussed below). It is convenient to take the upper limit of integration as infinite, since (a) the integral converges and the result is quite simple, and (b) if the dissipation range is important to the time scale we are likely in a regime where $\tau_{d}<\tau_{u}$. We therefore currently avoid the need to explicitly model the high-wavenumber spectrum. However, this may be revisited in the future if deemed necessary. Note that combining Eq. 8 with Eq. 10 leads to $\tau_{u} \sim \Delta^{2 / 3}$ which explains the two thirds scaling mentioned above and shown in Fig. 1.

For closure we require a model for the dissipation rate which is a physical parameter of the flow. The ideal model, therefore, does not vary with filter width (remember, the filter width is a user-specified artificial parameter). The model we employ assumes that production of subgrid-scale kinetic energy is equal to the dissipation of total kinetic energy [1],

$\varepsilon=\left(\mu_{t} / \bar{\rho}\right)|\tilde{S}|^{2}$,

where $|\tilde{S}| \equiv\left(2 \tilde{S}_{i j} \tilde{S}_{i j}\right)^{1 / 2}$ is the filtered rate of strain invariant. Pope [1] discusses the important observation that $|\tilde{S}| \sim \Delta^{-2 / 3}$ for $\Delta$ in the inertial subrange of an incompressible flow. For this limiting case, the constant coefficient Smagorinsky model gives $\left(\mu_{t} / \bar{\rho}\right)=\left(C_{s} \Delta\right)^{2}|\tilde{S}| \sim \Delta^{4 / 3}$ and we find the dissipation rate is correctly invariant with filter width, $\varepsilon \sim \Delta^{4 / 3}\left(\Delta^{-2 / 3}\right)^{2} \sim \Delta^{0}$.

\section{Effect of Buoyancy on the Dissipation Rate}

The turbulent viscosity is a critical component of the model: it affects large scale dynamics and energy dissipation, it affects diffusive transport, and it affects the rate of reaction in the EDC combustion model. As with the mixing rate, it is important that we consider the behavior of the turbulent viscosity as the filter width varies. Most LES models assume the filter width lies within the inertial subrange. If this is the case we want to use the dynamic Smagorinsky model $[12,13,18]$. If the flow is under-resolved, however, the dynamic model may lead to wildly oscillatory behavior. For consideration, here we propose a viscosity model to deal with under-resolved, buoyant flows that retains the desired convergent behavior of the dynamic model. A turbulent viscosity based on the graviational time scale, similar to Teiszen's model [19], may be constructed as follows,

$\mu_{t}= \begin{cases}\mu_{\text {Smag }}=\bar{\rho}\left(C_{s}[\mathbf{x}, t] \Delta\right)^{2}|\tilde{S}| & \text { if } \quad \Delta \leq \Delta_{g} \\ \mu_{\text {Grav }}=\frac{\Delta^{3}}{\operatorname{Pr}_{t} \tau_{g}} \max (0, \partial \bar{\rho} / \partial z) & \text { if } \quad \Delta>\Delta_{g}\end{cases}$

where, employing Eq. 9, the gravitational length scale is

$\Delta_{g}=\frac{2}{g}\left(\frac{\operatorname{Pr}_{t} C_{s}^{2} \bar{\rho}|\tilde{S}|}{\max (0, \partial \bar{\rho} / \partial z)}\right)^{2}$.

Equation 13 is derived by equating the viscosities $\mu_{\text {Smag }}$ and $\mu_{\text {Grav }}$ with $C_{s}=0.2$ and has the effect of automatically selecting the flow regime. If the density stratification is neutral or stable $(\partial \bar{\rho} / \partial z \leq 0)$, then $\Delta_{g}=\infty$ and we employ the dynamic Smagorinsky model. On the other hand, if the cell size is large relative the to gravitational length scale, this implies that the unstable stratification will dominate momentum transport and subgrid kinetic energy production. When the gravitational viscosity is invoked, the LES dissipation rate (i.e. production of subgrid kinetic energy) becomes 
$\varepsilon=\frac{\mu_{\mathrm{Smag}}}{\bar{\rho}^{2} \operatorname{Pr}_{t} C_{s}^{2} \sqrt{2}} \sqrt{g \Delta}|\tilde{S}| \max (0, \partial \bar{\rho} / \partial z)$.

Equation 14 is similar to a typical $k$ - $\varepsilon$ RANS model for production of turbulent kinetic energy due to buoyancy (see $[19,20]$ ), except here the acceleration scale, typcially just $g$, is given by $\sqrt{g \Delta}|\tilde{S}|$, highlighting the interplay between gravity and strain. Of further note, the quantity $\mu_{\text {Smag }} / C_{s}^{2}$ corresponds to an effective viscosity from a $k$ - $\varepsilon$ model, acceptable since the LES viscosity is usually less than that for RANS. Lastly, note that Eq. 14 is unlikely to directly enter into $\tau_{m i x}$ since if $\Delta>\Delta_{g}$ we are likely in a regime where $\tau_{g}<\tau_{u}$ (use of the dynamic model makes this difficult to analyze a priori).

Based on preliminary tests (not shown here), the model proposed in Eq. 12 performs quite well for extremely coarse grid resolution, even generating realistic puffing modes for what would normally become a stationary flame if simulated with constant coefficient Smagorinsky. Ultimately, however, we were unable to get this model to interact well with the dynamic Smagorinsky model for refined calculations. We speculate that this is because we did not include the gravitational viscosity in the dynamic procedure. Future work will consider this possibility. All results presented later in this paper are performed with dynamic Smagorinsky only.

\section{RESULTS}

\section{Heskestad Flame Height Correlation}

In this section, we compare FDS flame height predictions using the proposed new reaction model with the Heskestad correlation [21] given by

$\frac{L_{f}}{D}=3.7\left(Q^{*}\right)^{2 / 5}-1.02$

where $L_{f}$ is the flame height, $D$ is the effective diameter of the base of the plume, and $Q^{*}$ is the fire Froude number,

$Q^{*}=\frac{\dot{Q}}{\rho_{\infty} c_{p} T_{\infty} \sqrt{g D} D^{2}}$.

Ambient values for density, specific heat, and temperature are taken to be $\rho_{\infty}=1.20 \mathrm{~kg} / \mathrm{m}^{3}, c_{p}=1.01 \mathrm{~kJ} / \mathrm{kg} \cdot \mathrm{K}$, and $T_{\infty}=293 \mathrm{~K}$, respectively. The global heat release of the plume is $\dot{Q}(\mathrm{~kW})$ and the gravitational acceleration is $g=9.81 \mathrm{~m} / \mathrm{s}^{2}$. It is also useful to define the length scale

$D^{*}=\left(Q^{*}\right)^{2 / 5} D$.

Table 1 lists the parameters for 16 FDS calculations of a propane fire in a $1 \mathrm{~m}$ by $1 \mathrm{~m}$ square pan (the effective diameter is $D=1.13 \mathrm{~m}$, obtained by equating the area of a square and a circle). The heat release rate used in the FDS input file is obtained for each $Q^{*}$ case using Eq. 16. Three uniform mesh resolutions are tested corresponding to $D^{*} / \delta x=5,10$, and 20. Note that the model filter width is taken as the mesh spacing. The domain set up for each resolution depends on $D^{*}$, but the number of grid points for a given $D^{*} / \delta x$ remains fixed. For the coarsest resolution, $N_{x} \times N_{y} \times N_{z}=16 \times 16 \times 40$ ( $z$ is the vertical direction). Note that all the input files used for the test cases presented here may be downloaded from the FDS repository (http://fire.nist.gov/fds) under the directory Validation/Flame_Height/FDS_Input_Files. FDS SVN (subversion number) 7707 was used for all calculations.

The flame height for the FDS simulations is defined as the distance above the pan, on average, at which 99\% of the fuel has been consumed. At 1000 time increments within each run, FDS averages the heat release in a horizontal plane and reports the heat release per unit length (HRRPUL) for each increment in $z$. Each case is run for roughly 50 puffing cycles. In a post-processing step, the HRRPUL values are time averaged over the last half of the simulation and integrated in the vertical direction until the sum reaches $99 \%$ of the $\dot{Q}$ value used in the input file. 
Table 1. Summary of case parameters.

\begin{tabular}{|c|c|c|}
\hline$Q^{*}$ & $\dot{Q}(\mathrm{~kW})$ & $D^{*}(\mathrm{~m})$ \\
\hline \hline 0.1 & 151 & 0.45 \\
\hline 0.2 & 303 & 0.59 \\
\hline 0.5 & 756 & 0.86 \\
\hline 1 & 1513 & 1.13 \\
\hline 2 & 3025 & 1.49 \\
\hline 5 & 7564 & 2.15 \\
\hline 10 & 15127 & 2.84 \\
\hline 20 & 30255 & 3.75 \\
\hline 50 & 75636 & 5.40 \\
\hline 100 & 151273 & 7.13 \\
\hline 200 & 302545 & 9.41 \\
\hline 500 & 756363 & 13.6 \\
\hline 1000 & 1512725 & 17.9 \\
\hline 2000 & 3025450 & 23.6 \\
\hline 5000 & 7563625 & 34.1 \\
\hline 10000 & 15127250 & 45.0 \\
\hline
\end{tabular}

Since the data underlying the Heskestad correlation were gathered visually from time exposed flame images, the flame height definitions are clearly a potential source of error. For this work, we limit our comparisons to a low sooting fuel (propane) to avoid issues related to smoke obscuration in data collection. The effect of highly sooting fuels on the heat flux distribution and flame dynamics is beyond the scope of this article. For the current set of results, the default radiation model in FDS is employed. In short, absorption is based on a diffuse gray gas approximation and emission in the flame region is based on a constant radiant fraction $\chi_{r}=0.35$ times the local heat release per unit volume.

Figure 2 shows the results for FDS flame height predictions with the new reaction model. The results are improved over previous studies [22]. The log-log plot makes it difficult to appreciate the magnitude of the differences between the FDS predictions and the correlation. Considering all three grid resolutions, the average relative error is $7.3 \%$ for cases with $Q^{*} \geq 1$ and $24 \%$ for the cases where $Q^{*}<1$. This trend is commensurate with the spread in the correlation predictions compiled by McCaffrey [21]. For the cases shown here, therefore, the FDS predictions fall within the expected uncertainty of a hand calculation method (hopefully a minimum requirement for a CFD result). To give the reader a qualitative feel for the results, Fig. 3 shows instantaneous flame images for the $Q^{*}=1$ case at coarse, medium, and fine resolutions. The flame surface is represented by a heat release threshold.

\section{Discussion}

One may now question which aspect of the new model is responsible for the improved results. How do we know which time scale controls the mixing in a given case? To answer this question, we first remark that during the development of the model we used print statements within the reaction subroutine to compare all the time scales locally and indeed the picture presented in Fig. 1 does emerge. However, for more direct evidence, Fig. 4 shows flame height predictions for the coarse resolution set up with two different time scale models. In the first, the mixing time is simply set equal the to subgrid advection time scale, $\tau_{m i x}=\tau_{u}$ (stars). The flame heights are clearly too large. In fact, these heights are near the upper boundary of the computational domain. In the second set of results, the reaction time scale is set equal to the acceleration time scale, $\tau_{\text {mix }}=\tau_{g}$ (squares). The results are quite good, though slightly too high. We may infer that at coarse resolution the acceleration time scale is generally controlling, but by revisiting the coarse results in Fig. 2, which are better than the $\tau_{g}$ results in Fig. 4, we may also infer that in the complete model the advective time scale does play a role locally in shortening the flame height (consuming fuel) for coarse simulations.

One of the reasons why the acceleration time scale improves results for the high $Q^{*}$ cases, which are practically jet flames, may be related to the jet exit boundary condition which is grossly under-resolved. Consider 


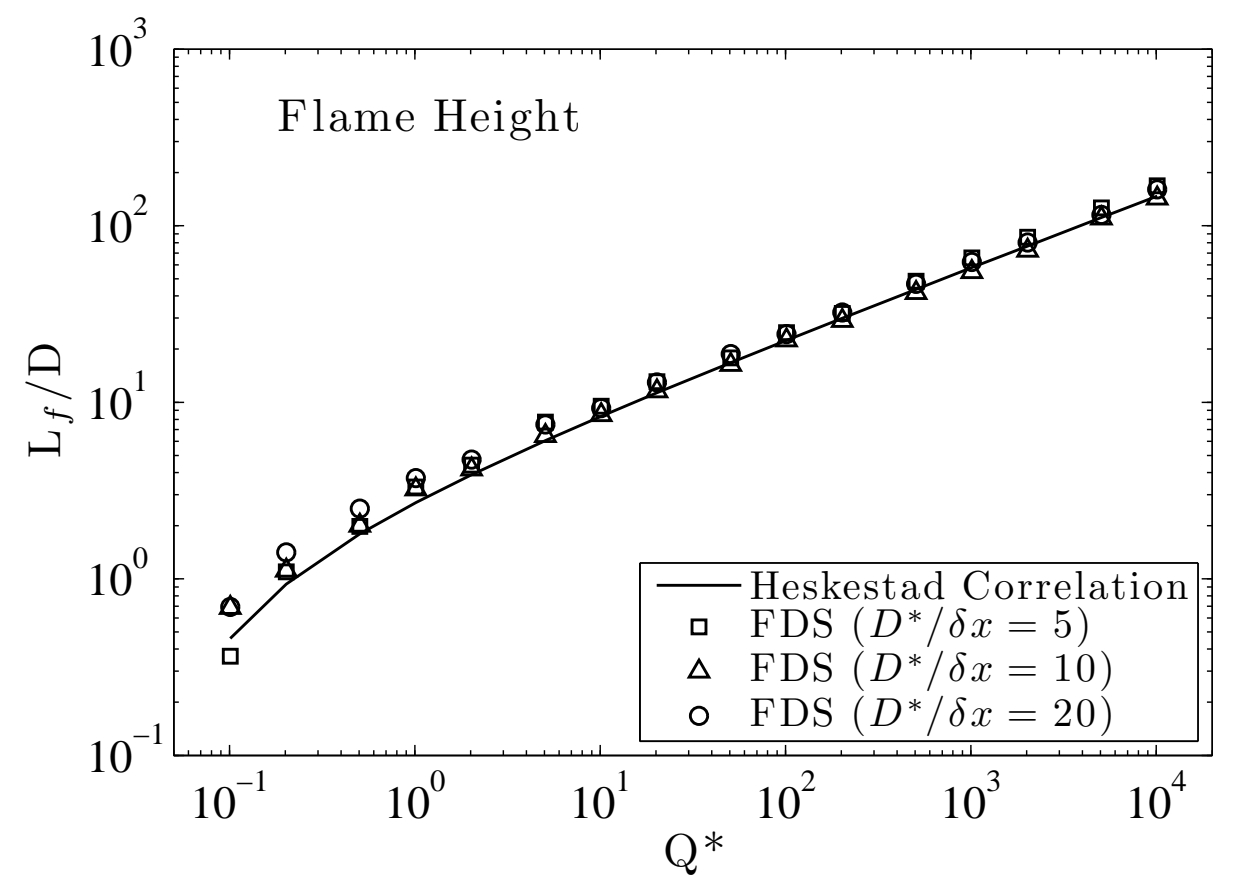

Fig. 2. FDS predictions for flame height compared with Heskestad's correlation.

the $Q^{*}=10^{4}$ case, for example. Referring to Table 1 , note that the coarse grid spacing is $\delta x=D^{*} / 5=9 \mathrm{~m}$. Therefore, the fuel mass flow from the $1 \mathrm{~m}^{2}$ pan is smeared out over 81 square meters! The mean advection velocity is hopelessly too low to deliver an accurate description of the mixing. However, while the local velocity may be in error, results from the new model suggest that the local acceleration due to gravity may still be a useful quantity.

The inaccuracy for the low $Q^{*}$ cases may be explained in two ways. First, it is important to resolve both the base and height of the flame, and when $Q^{*}<1$ the height is under-resolved compared to the base. While it is reasonable to expect the modeler to add cells in the vertical direction, in practice we often do not know the heat release a priori and coarse, cubic cells are required for tractable computations. Until adaptive mesh methods are available, a second approach to improving accuracy at low $Q^{*}$ is to develop improved turbulence models, as attempted with Eq. 12.

The next logical step in development of the time scale model might be to generalize the acceleration to include other forces. This would in principle allow the model to address momentum dominated flows at coarse resolution. Such generalization was explored but failed to improve the results for typical buoyancy driven fire applications (several cases in the FDS validation suite [6] are routinely tested during development). Given the simplicity and robustness of the current approach, the complications inherent in generalizing the acceleration do not seem warranted at the present time.

\section{CONCLUSIONS}

This work addresses the problem of specifying the reaction time scale for coarsely resolved fire dynamics simulations. We build upon well-understood principles in turbulence modeling and extend the eddy dissipation concept (fast chemistry) time scale model to consider the limiting case where the fuel load is fully consumed within a single grid cell. This leads to a proposal to connect the turbulent advection mixing regime with the flame scale regime via a mixing time scale based on buoyant acceleration.

The new model is tested by comparing simulations at three different grid resolutions with the Heskestad flame height correlation [21]; a total of 48 cases are run. The results are reasonably insensitive to grid resolution and the errors relative to the correlation fall within experimental uncertainty. 

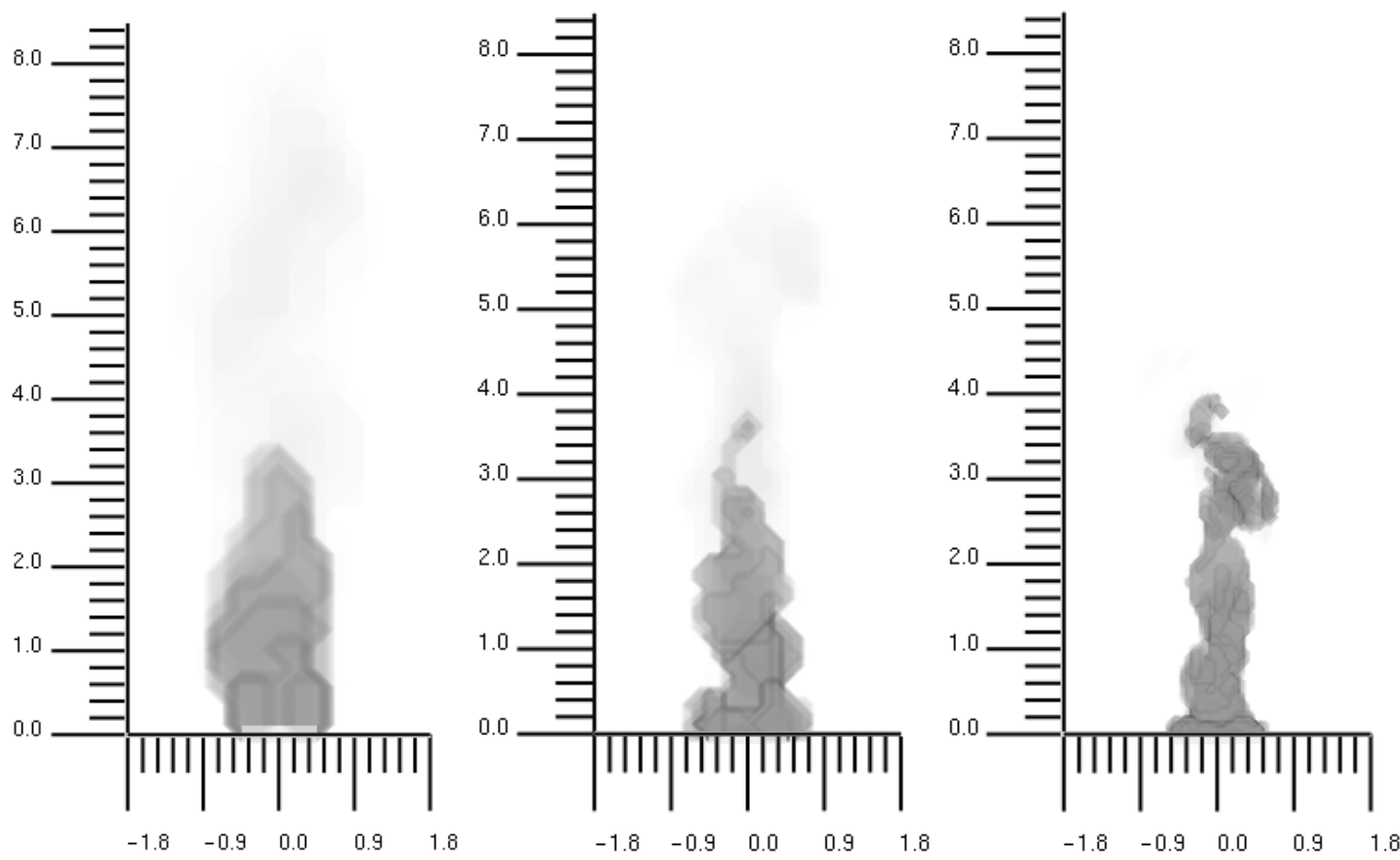

Fig. 3. Instantaneous flame images from $Q^{*}=1 \mathrm{FDS}$ calculations, from left to right, for coarse, medium, and fine grid resolutions. The flame surface is represented by a heat release threshold. Dimensions are in meters.

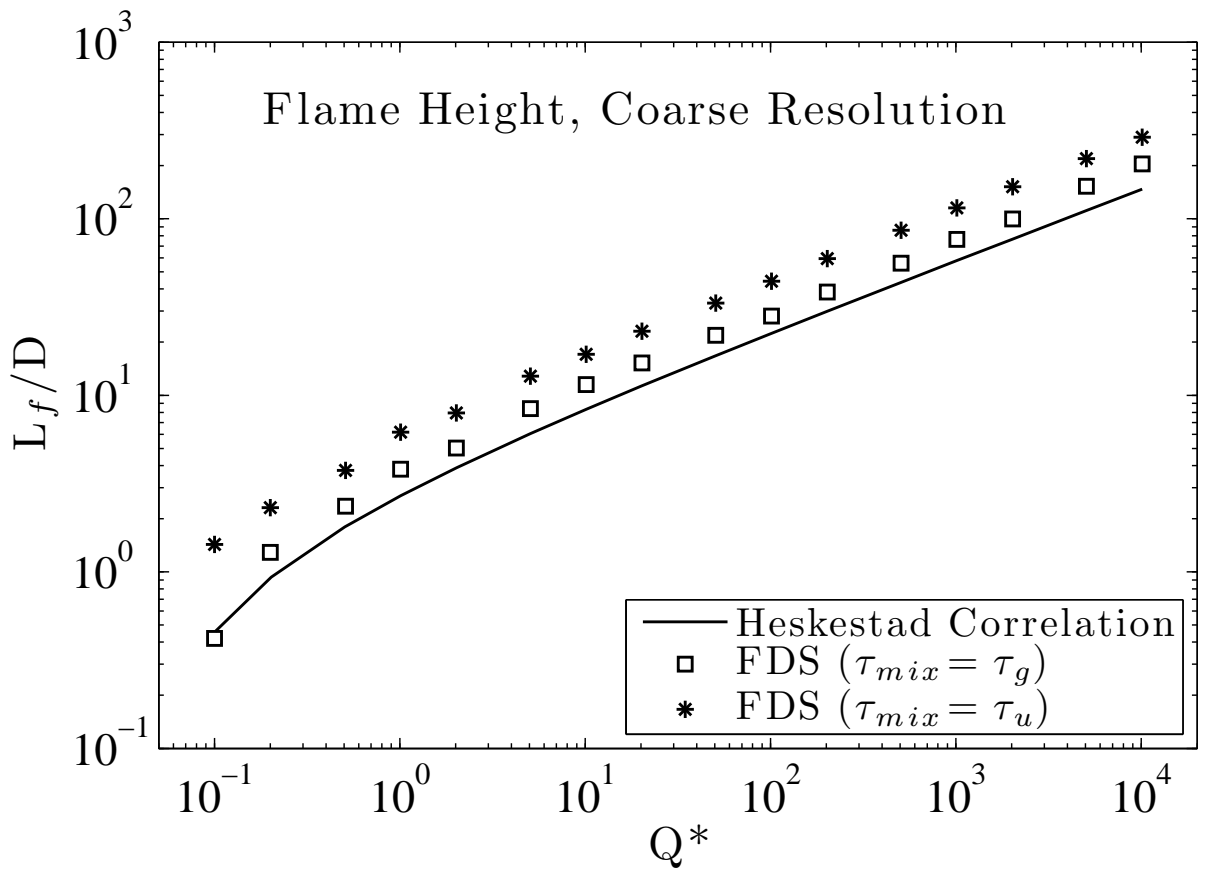

Fig. 4. Comparison of $D^{*} / \delta x=5$ results with two different reaction time scales.

In the future we plan to test the proposed model on all the cases available in the FDS validation suite [6] and to continue development of the model proposed in Eq. 12 which holds promise for extremely coarse grids. The performance of the new reaction model in predictions of heat flux distribution is of particular interest. But 
such problems may also strongly depend on the submodels for soot formation and convective and radiative heat transfer, which require independent validation. As we move forward in development of physics-based fire models, we should heed the overarching theme of the present work, which is to consider the performance of submodels across a broad range of length scales and to seek simple scaling laws to connect well-understood limiting cases.

\section{ACKNOWLEDGEMENTS}

The authors would like to thank the US Nuclear Regulatory Commission and the US Forest Service for their financial support. Additionally, the authors are indebted to John de Ris of FM Global for discussions which inspired the ideas in this work. Further insights are owed to Alan Kerstein of Sandia National Laboratories, Livermore.

\section{REFERENCES}

[1] Pope, S. B. Turbulent Flows. Cambridge, 2000.

[2] Pope, S. B. Ten questions concerning the large-eddy simulation of turbulent flows. New Journal of Physics, 6:1-24, 2004, http://dx.doi.org/10.1088/1367-2630/6/1/035.

[3] Meyers, J., Geurts, B., and Sagaut, P., (ed.). Quality and Reliability of Large-Eddy Simulations. Springer, 2008.

[4] McGrattan, K., Hostikka, S., Floyd, J., Baum, H., Rehm, R., Mell, W., and McDermott, R. Fire Dynamics Simulator (Version 5), Technical Reference Guide, Volume 1: Mathematical Model. NIST Special Publication 1018-5, National Institute of Standards and Technology, Gaithersburg, Maryland, September 2010.

[5] McDermott, R., McGrattan, K., Hostikka, S., and Floyd, J. Fire Dynamics Simulator (Version 5), Technical Reference Guide, Volume 2: Verification. NIST Special Publication 1018-5, National Institute of Standards and Technology, Gaithersburg, Maryland, September 2010.

[6] McGrattan, K., Hostikka, S., Floyd, J., and McDermott, R. Fire Dynamics Simulator (Version 5), Technical Reference Guide, Volume 3: Validation. NIST Special Publication 1018-5, National Institute of Standards and Technology, Gaithersburg, Maryland, September 2010.

[7] Fox, R. O. Computational Models for Turbulent Reacting Flows. Cambridge, 2003.

[8] Peters, N. Turbulent Combustion. Cambridge, 2000.

[9] Poinsot, T. and Veynante, D. Theoretical and Numerical Combustion (2nd ed.). Edwards, 2005.

[10] Magnussen, B. F. and Hjertager, B. H. On mathematical modeling of turbulent combustion with special emphasis on soot formation and combustion. In 16th Symp. (Int.) on Combustion, Pittsburgh, 1976, http://dx.doi.org/10.1016/S0082-0784(77)80366-4. The Combustion Institute.

[11] Ertesvåg, I. S. and Magnussen, B. F. The eddy dissipation turbulent energy cascade model. Comb. Sci. Tech., 2001, http://dx.doi.org/10.1080/00102200008935784.

[12] Germano, M., Piomelli, U., Moin, P., and Cabot, W. A dynamic subgrid-scale eddy viscosity model. Phys. Fluids A, 3(7):1760-1765, 1991, http://dx.doi.org/10.1063/1.857955.

[13] Moin, P., Squires, K., Cabot, W., and Lee, S. A dynamic subgrid-scale model for compressible turbulence and scalar transport. Phys. Fluids A, 3(11):2746-2757, 1991, http://dx.doi.org/10.1063/ 1.858164 .

[14] Roe, P. L. Characteristics-based schemes for the Euler equations. Ann. Rev. Fluid Mech., 18:337, 1986, http://dx.doi.org/10.1146/annurev.fl.18.010186.002005. 
[15] Zhang, W., Ryder, N., Roby, R. J., and Carpenter, D. Modeling of the combustion in compartment fires using large eddy simulation approach. In Proceedings of the 2001 Fall Technical Meeting, Eastern States Section, Pittsburgh, Pennsylvania, USA, 2005. The Combustion Institute.

[16] Vaari, J., Floyd, J., and McDermott, R. CFD simulations on extinction of co-flow diffusion flames. In Fire Safety Science - Proceedings of the 10th International Symposium, University of Maryland, College Park, Maryland, USA, 2011. International Association for Fire Safety Science.

[17] Beyler, C. Flammability Limits of Premixed and Diffusion Flames. SFPE Handbook of Fire Protection Engineering (4th ed.), DiNenno, P. J. (ed.), National Fire Protection Association, Quincy, Massachusetts 02269, 2008, p. 2/194.

[18] Smagorinsky, J. General Circulation Experiments with the Primitive Equations. I. The Basic Experiment. Monthly Weather Review, 91(3):99-164, 1963, http://dx.doi.org/10.1175/15200493(1963)091<0099:GCEWTP>2.3.CO;2.

[19] Teiszen, S. R., Pitsch, H., Blanquart, G., and Abarzhi, S. Toward the development of a LES-SGS closure model for buoyant plumes. Stanford Center for Turbulence Research, Proceedings of the Summer Program, pp. 341-352, 2004.

[20] Merci, B. and Van Maele, K. Influence of the turbulence model in numerical simulations of fire in a ventilated horizontal tunnel. In Proceedings of the European Combustion Meeting, 2005.

[21] Heskestad, G. Fire Plumes, Flame Height and Air Entrainment. SFPE Handbook of Fire Protection Engineering (4th ed.), DiNenno, P. J. (ed.), National Fire Protection Association, Quincy, Massachusetts 02269, 2008, p. $2 / 1$.

[22] McGrattan, K., Floyd, J., Forney, G., Baum, H., and Hostikka, S. Improved radiation and combustion routines for a large eddy simulation fire model. In Fire Safety Science-Proceedings of the 7th International Symposium, pp. 827-838, Worcester Polytechnic Institute, Worscester, Massachusetts, USA, 2002, http://dx.doi.org/10.3801/IAFSS.FSS.7-827. International Association for Fire Safety Science. 